\title{
Prevalencias de bajo peso y pequeño para la edad gestacional en Argentina: comparación entre el estándar INTERGROWTH-21 ${ }^{\text {st }} y$ una referencia argentina
}

\author{
Prevalence of underweight and small for gestational age in \\ Argentina: Comparison between the INTERGROWTH-21 ${ }^{\text {st }}$ \\ standard and an Argentine reference
}

\author{
Lic. Gabriela B. Revollo ${ }^{a}$, Lic. Jorge I. Martínez ${ }^{a}$, Dr. Carlos Grandi ${ }^{b}$ \\ Dra. Emma L. Alfaro y yr. José E. Dipierria
}

a. Instituto de Ecorregiones Andinas (INECOA), Consejo Nacional de Investigaciones Científicas y Técnicas (CONICET), Universidad Nacional de Jujuy (UNJu), Instituto de Biología de la Altura (INBIAL), San Salvador de Jujuy.

b. Profesor visitante, Fac. Medicina, Univ. San Pablo, Brasil.

Correspondencia:

Lic. Gabriela B. Revollo, gabrielarevollo@gmail.com

Financiamiento: El artículo está enmarcado y financiado por el proyecto Perfil Antropométrico y Altura Geográfica en Poblaciones Infantojuveniles Jujeñas, Secretaría de Ciencia y Técnica y Estudios RegionalesUniversidad Nacional de Jujuy, SECTERUNJu (período 20162019).

Conflicto de intereses: Ninguno que declarar.

Recibido: 8-2-2017 Aceptado: 31-5-2017

Publicado Primero en Internet: 13-10-2017

\section{RESUMEN}

Introducción. Bajo peso al nacer $(<2500$ g) incluye recién nacidos pretérmino y a término pequeños para la edad gestacional $(\mathrm{PEG})(<\mathrm{P} 10)$. La Organización Mundial de la Salud define bajo peso (BP) como peso al nacer $<\mathrm{P} 3$ peso/edad. Internacionalmente, no existe consenso sobre estándares y/o referencias de peso al nacer por edad gestacional (EG) para evaluar PEG y BP en pretérminos. Se determinó la prevalencia de BP y PEG con el estándar INTERGROWTH-21 ${ }^{\text {st }}$ y la referencia poblacional argentina de Urquía, y se analizó la concordancia de las prevalencias entre ambas herramientas.

Población y métodos. Estudio observacional, analítico y retrospectivo realizado sobre los nacimientos registrados en 2013 en el Ministerio de Salud de la Nación. Los criterios de exclusión fueron EG $<24^{+0}->42^{+6}$ semanas, embarazo gemelar y ausencia de datos de peso, EG y sexo. Se calcularon las prevalencias por sexo, regiones y categorías de prematurez de BP y PEG con el estándar y la referencia. La concordancia se evaluó con Kappa.

Resultados. Las prevalencias de BPy PEG fueron más altas con el estándar en pretérmino; lo contrario se observó en recién nacidos a término. La significación estadística varió según categorías deEG, sexo y regiones. Las prevalencias más altas se presentaron en regiones del norte argentino y las concordancias entre prevalencias oscilaron entre débiles y muy buenas.

Conclusiones. Las concordancias de prevalencias de BP y PEG obtenidas con el estándar y la referencia en pretérmino y a término fueron moderadas, y se observó variabilidad interregional. Los resultados plantean nuevas perspectivas auxológicas en la evaluación epidemiológica del retardo del crecimiento intrauterino en Argentina.

Palabras clave: gráficos de crecimiento, retardo del crecimiento fetal, recién nacido pequeño para la edad gestacional, prevalencia.

http:/ / dx.doi.org/10.5546/ aap.2017.547

Texto completo en inglés:

http:/ / dx.doi.org/10.5546/ aap.2017.eng.547

Cómo citar: Revollo GB, Martínez JI, Grandi C, et al. Prevalencias de bajo peso y pequeño para la edad gestacional en Argentina: comparación entre el estándar INTERGROWTH-21 $1^{\text {st }}$ y una referencia argentina. Arch Argent Pediatr 2017;115(6):547-555.

\section{INTRODUCCIÓN}

La prevalencia de bajo peso al nacer (BPN, peso al nacer [PN] $<2500$ g) constituye un indicador general de salud, ilustrativo de las circunstancias socioeconómicas y ambientales del individuo y la sociedad. ${ }^{1}$ A través de la Resolución 65.6, la Asamblea Mundial de la Salud propone reducir en un $30 \%$ el BPN para $2025 .^{2}$

EL BPN incluye a niños pequeños para la edad gestacional (PEG) y pretérminos, y la superposición de ambas condiciones. PEG es un indicador de retardo del crecimiento intrauterino y, conjuntamente con la prematurez, constituye un factor de riesgo de mortalidad fetal, neonatal e infantil y consecuencias negativas para la salud a largo plazo. ${ }^{3,4}$

No existe consenso internacional respecto al análisis antropométrico en pretérminos. El Comité Nacional de Crecimiento y Desarrollo y el Comité de Estudios Fetoneonatales proponen las curvas de Fenton y Kim para el seguimiento del recién nacido (RN) prematuro. ${ }^{5}$ Las referencias describen cómo los sujetos "han crecido" en un momento y lugar en particular; en cambio, los estándares son prescriptivos y describen cómo los sujetos "deberían crecer" en condiciones óptimas. Recientemente, se publicaron los estándares de peso, talla y circunferencia craneana para $\mathrm{RN}$ por sexo y edad gestacional (EG) del International Fetal and Newborn 
Growth Consortium for the $21^{\text {st }}$ Century Project (INTERGROWTH-21 ${ }^{\text {st }}$ ). ${ }^{6}$ Se trata de un estudio transversal, multicéntrico y transcultural del crecimiento del $\mathrm{RN}$, realizado con el mismo enfoque prescriptivo y diseño metodológico que el utilizado para la producción de los estándares de evaluación del crecimiento infantojuvenil de la Organización Mundial de la Salud (OMS) vigentes en Argentina. ${ }^{7,8}$ INTERGROWTH-21 ${ }^{\text {st }}$ posibilita el análisis antropométrico del RN a término y pretérmino entre las $24^{+0}$ y las $42^{+6}$ semanas de EG. En 2011, Urquía et ál. publicaron una referencia poblacional argentina del PN, representativa de la población argentina reciente, ya que incluyó todos los nacimientos entre 2003 y $2007 .{ }^{9}$

La OMS define bajo peso (BP) como el PN para la EG $<$ P3 del estándar WHO Child Growth Standards, ${ }^{7}$ que resulta apropiado para los RN a término en los que la EG no es fiablemente conocida y para los que no presentan BPN. Cuando la EG es conocida con precisión y los RN presentan retardo del crecimiento, es preferible utilizar un estándar o una referencia apropiada de PN por EG. Villar et al., ${ }^{10}$ definen 2 fenotipos distintos del crecimiento fetal alterado, análogos a los propuestos por la OMS, para definir malnutrición en RN: acortado y emaciado. La definición de estos fenotipos se basa en las mediciones de la longitud (acortamiento) y del índice de masa corporal (emaciación) al nacer menores del P3 de INTERGROWTH-21 ${ }^{\text {st }}$. Por extensión, en este trabajo, se utiliza BP para describir malnutrición fetal.

El objetivo fue determinar la prevalencia de BP y PEG por sexo a nivel regional por EG en $\mathrm{RN}$ argentinos con el estándar INTERGROWTH$21^{\text {st } 6}$ y la referencia poblacional argentina de Urquía, ${ }^{9}$ y analizar la concordancia entre ambas prevalencias.

\section{POBLACIÓN Y MÉTODOS}

Estudio observacional, analítico y retrospectivo realizado sobre todos los nacimientos vivos de Argentina en 2013. Los datos, de acceso libre, fueron obtenidos del Informe Estadístico de Recién Nacidos (Dirección de Estadística e Información de Salud, Ministerio de Salud). ${ }^{11}$ Los criterios de exclusión fueron EG $<24^{+0}>42^{+6}$ semanas, embarazo gemelar y falta de datos de peso, EG y sexo.

La restricción del crecimiento fetal se clasificó según los siguientes indicadores: $\mathrm{BP}(<\mathrm{P} 3 \mathrm{PN} /$ EG) y PEG (< P10 PN/EG). Para definir BP y
PEG, se utilizó el estándar INTERGROWTH-21 $1^{\text {st }}$ y la referencia poblacional argentina de Urquía de PN. ${ }^{9}$

Según la EG, los RN se agruparon en las siguientes categorías: a) prematuros extremos $\left(<28^{+0}\right.$ semanas $) ;$ b) muy prematuros $\left(28^{+0}-\leq 31^{+6}\right.$ semanas $)$; c) prematuros de moderados a tardíos $\left(32^{+0}-\leq 36^{+6}\right.$ semanas); y d) término $\left(\geq 37^{+0}\right.$ semanas). ${ }^{12}$

Las prevalencias de BP y PEG se calcularon por sexo y categorías de EG por regiones censales: 1) Noroeste Argentino (NOA) (Jujuy, Salta, Tucumán, Santiago del Estero, Catamarca y La Rioja); 2) Noreste Argentino (NEA) (Formosa, Chaco, Misiones y Corrientes); 3) Cuyo (San Juan, San Luis y Mendoza); 4) Centro (Santa Fe, Córdoba, Entre Ríos, Buenos Aires y La Pampa); 5) Ciudad Autónoma de Buenos Aires (CABA); y 6) Patagonia (Neuquén, Río Negro, Chubut, Santa Cruz y Tierra del Fuego). Además, se calculó la prevalencia regional de BPN.

Se realizaron comparaciones gráficas entre los P3 y P10 de INTERGROWTH-2 ${ }^{\text {st }}$ y la referencia poblacional argentina de Urquía. Se utilizó el índice Kappa para evaluar la concordancia entre las prevalencias, que se clasificó en pobre $(\leq 0,20)$, débil $(0,21-0,40)$, moderada $(0,41-0,60)$, buena $(0,61-0,80)$ y muy buena $(>0,80) \cdot{ }^{13}$ Las diferencias de prevalencias entre sexos y según la referencia y el estándar fueron calculadas con la prueba de $\mathrm{Ji}^{2}$. El nivel de significación se fijó en $\mathrm{p}<0,001$ por el elevado tamaño muestral y se emplearon los programas SPSS IBM versión 22 y MEDCALC.

\section{RESULTADOS}

La población incluyó a $735491 \mathrm{RN}$ vivos de Argentina en 2013. En la Figura 1 y 2, se presentan los P3 y P10 del PN por sexo empleando INTERGROWTH-21 ${ }^{\text {st }}$ y la referencia poblacional argentina de Urquía. En ambos sexos, desde las semanas $27^{+0}-33^{+6}$, ambos percentilos son más altos empleando INTERGROWTH-21 ${ }^{\text {st; }}$ desde las $33^{+0}$ hasta las $36^{+6}$ semanas, los percentilos de la referencia y el estándar, prácticamente, se superponen, $\mathrm{y}$, a partir de la semana $37^{+0}$, la referencia presenta percentilos más altos.

En las Tablas 1 y 2, se presentan las prevalencias de BP y PEG por sexo y categorías de EG. En ambos sexos, las prevalencias de BP y PEG fueron más altas con el estándar en todas las categorías de prematurez; lo contrario se observó en los RN a término. La significación estadística de estas diferencias fue muy heterogénea según categorías de EG, sexo y regiones geográficas, pero, de 
manera llamativa, la categoría término presentó diferencias estadísticamente significativas en todas las regiones y en ambos sexos. En las categorías muy prematuro y prematuro de moderado a tardío, en algunas regiones y, exclusivamente, en varones, la concordancia entre las prevalencias de BP y PEG en ambos sexos fueron muy buenas $(>0,80)$, mientras que, en las restantes categorías y en los $\mathrm{RN}$ a término, las concordancias oscilaron entre regular y buena.
En la Figura 3, se presenta la distribución por regiones de las prevalencias de BPN, BP y PEG de acuerdo con el estándar y con la referencia independientemente del sexo. Mientras la prevalencia de BPN es semejante entre las regiones, se observa una mayor heterogeneidad interregional de BP y PEG. CABA y Patagonia exhibieron las prevalencias más bajas de ambos indicadores tanto con la referencia como con el estándar.

FIGURA 1: Comparación entre la referencia poblacional argentina de Urquía y el estándar Intergrowth-21 ${ }^{\text {st }}$ de los percentilos 3 y 10 del peso de nacimiento (Argentina, 2013, varones)

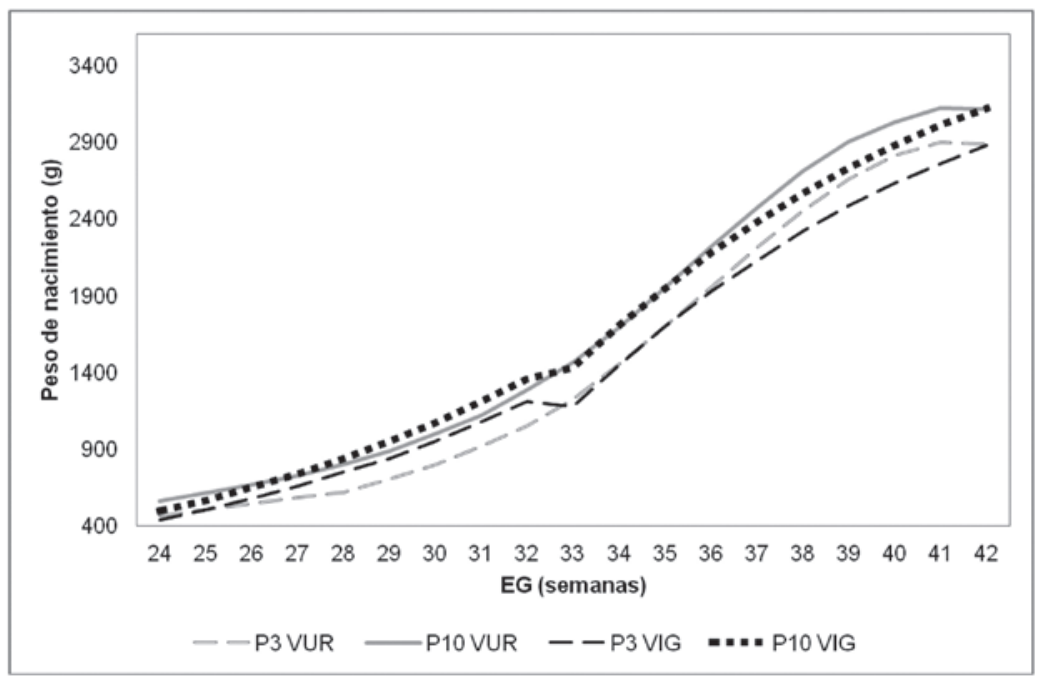

EG: edad gestacional. P3 VUR: percentilo 3, varones, Urquía et al. P10 VUR: percentilo 10, varones, Urquía et al. P3 VIG: percentilo 3, varones, INTERGROWTH-21 $1^{\text {st }}$ P10 VIG: percentilo 10, varones, INTERGROWTH-21 ${ }^{\text {st }}$.

FIGURA 2: Comparación entre la referencia poblacional argentina de Urquía y el estándar Intergrowth-21 ${ }^{\text {st }}$ de los percentilos 3 y 10 del peso de nacimiento (Argentina, 2013, mujeres)

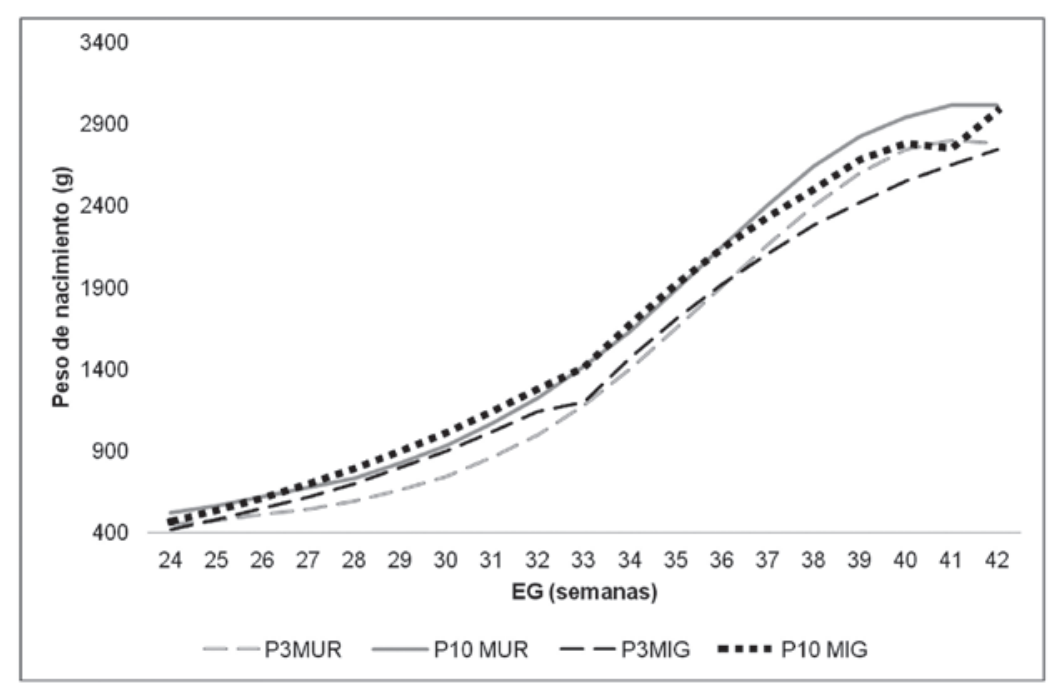

EG: edad gestacional. P3 MUR: percentilo 3, mujeres, Urquía et al. P10 MUR: percentilo 10, mujeres, Urquía et al. P3 MIG: percentilo 3, mujeres, INTERGROWTH-21 $1^{\text {st }}$ P10 MIG: percentilo 10, mujeres, INTERGROWTH-21 ${ }^{\text {st }}$. 
A nivel país, las prevalencias de BP fueron muy similares entre varones y mujeres empleando INTERGROWTH-2 $1^{\text {st }} \mathrm{y}$ más altas en pretérminos que la referencia poblacional argentina de Urquía y estadísticamente significativas para muy prematuro, prematuro moderado y término. La concordancia fue de buena a muy buena en tres de las cuatro categorías.
Además, para PEG, las prevalencias fueron mayores con INTERGROWTH-21 ${ }^{\text {st }}$ solamente en mujeres, mientras que el índice Kappa fue de bueno a muy bueno en todas las categorías de EG.

\section{DISCUSIÓN}

A nivel país y aplicando el nuevo estándar de PN INTERGROWTH-21 ${ }^{\text {st }}$, se observó un aumento

TABLA 1: Prevalencias de bajo peso con la referencia poblacional argentina de Urquía y el estándar INTERGROWTH-21 ${ }^{\text {st }}$ por sexo y categorías de edad gestacional, según regiones de Argentina (2013)

\begin{tabular}{|c|c|c|c|c|c|c|c|c|c|c|c|}
\hline \multirow{3}{*}{$\begin{array}{c}\text { Región/ } \\
\text { país }\end{array}$} & \multirow{3}{*}{$\begin{array}{c}\text { Catego- } \\
\text { rías } \\
\text { EG }\end{array}$} & \multicolumn{5}{|c|}{ Mujeres } & \multicolumn{5}{|c|}{ Varones } \\
\hline & & \multicolumn{2}{|c|}{ Referencia } & \multicolumn{2}{|c|}{ Estándar } & \multirow{2}{*}{ Kappa } & \multicolumn{2}{|c|}{ Referencia } & \multicolumn{2}{|c|}{ Estándar } & \multirow{2}{*}{ Kappa } \\
\hline & & $\mathbf{N}$ & Prev. & $\mathbf{N}$ & Prev. & & $\mathbf{N}$ & Prev. & $\mathbf{N}$ & Prev. & \\
\hline \multirow{4}{*}{ NOA } & $\mathrm{PE}$ & 2 & 1,5 & 5 & 3,6 & 0,562 & 2 & 1,5 & 4 & 3,0 & 0,660 \\
\hline & MP & 3 & 1,1 & 20 & 7,3 & 0,247 & 1 & 0,4 & 20 & $7,2^{*}$ & 0,089 \\
\hline & PM & 126 & 3,4 & 153 & 4,1 & $0,899^{* *}$ & 144 & 3,8 & 149 & 3,9 & $0,925^{\text {** }}$ \\
\hline & $\mathrm{T}$ & 1372 & 3,1 & 683 & $1,5^{*}$ & 0,658 & 1572 & 3,4 & 799 & $1,7^{*}$ & 0,666 \\
\hline \multirow{4}{*}{ NEA } & PE & 2 & 1,9 & 4 & 3,9 & 0,658 & 2 & 1,7 & 3 & 2,5 & 0,388 \\
\hline & $\mathrm{MP}$ & 3 & 1,3 & 14 & $6,1^{*}$ & 0,339 & 2 & 0,8 & 14 & $5,6^{*}$ & 0,239 \\
\hline & PM & 104 & 3,5 & 124 & 4,1 & $0,909^{* *}$ & 100 & 3,3 & 105 & 3,4 & $0,894^{* *}$ \\
\hline & $\mathrm{T}$ & 1396 & 4,1 & 745 & $2,2^{*}$ & 0,687 & 1435 & 4,0 & 728 & $2,0^{*}$ & 0,664 \\
\hline \multirow{4}{*}{ CUYO } & $P E$ & 1 & 1,2 & 2 & $2,4^{*}$ & 0,661 & 0 & SD & 1 & 1,1 & SD \\
\hline & MP & 3 & 2,0 & 8 & 5,3 & 0,532 & $\mathbf{0}$ & SD & 7 & $4,1^{*}$ & SD \\
\hline & PM & 51 & 3,2 & 63 & 4,0 & $0,891^{* *}$ & 46 & 2,9 & 48 & 3,0 & $0,956^{* *}$ \\
\hline & $\mathrm{T}$ & 883 & 3,3 & 398 & $1,5^{*}$ & 0,613 & 898 & 3,4 & 456 & $1,7^{*}$ & 0,666 \\
\hline \multirow{4}{*}{ CENTRO } & $\mathrm{PE}$ & 9 & 1,6 & 19 & 3,4 & 0,635 & 17 & 2,5 & 26 & 3,8 & 0,688 \\
\hline & MP & 24 & 1,8 & 71 & $5,4^{*}$ & 0,491 & 19 & 1,3 & 77 & $5,2^{*}$ & 0,383 \\
\hline & PM & 481 & 3,1 & 592 & $3,8^{*}$ & $0,893^{* *}$ & 590 & 3,4 & 615 & 3,5 & $0,917^{\star \star *}$ \\
\hline & $\mathrm{T}$ & 7154 & 3,9 & 4049 & $2,2^{*}$ & 0,715 & 7899 & 4,0 & 4429 & $2,2^{*}$ & 0,710 \\
\hline \multirow{4}{*}{ CABA } & $\mathrm{PE}$ & 0 & SD (?) & 1 & 1,6 & SD (?) & 4 & 4,4 & 5 & 5,6 & $0,883^{\text {*** }}$ \\
\hline & MP & 3 & 2,0 & 11 & 7,3 & 0,410 & 5 & 2,9 & 11 & 6,5 & 0,609 \\
\hline & PM & 36 & 2,6 & 44 & 3,1 & $0,897^{* *}$ & 47 & 3,1 & 51 & 3,4 & $0,895^{\text {***}}$ \\
\hline & $\mathrm{T}$ & 537 & 2,8 & 250 & $1,3^{*}$ & 0,629 & 524 & 2,6 & 223 & $1,1^{*}$ & 0,591 \\
\hline \multirow{4}{*}{$\begin{array}{l}\text { PATA- } \\
\text { GONIA }\end{array}$} & PE & 2 & 3,3 & 4 & 6,7 & 0,651 & 1 & 1,3 & 3 & 3,8 & 0,490 \\
\hline & MP & 1 & 0,8 & 7 & 5,5 & 0,240 & 5 & 2,7 & 9 & 4,9 & 0,704 \\
\hline & PM & 26 & 1,8 & 36 & 2,6 & $0,835^{* *}$ & 34 & 2,1 & 35 & 2,2 & $0,896^{\text {** }}$ \\
\hline & $\mathrm{T}$ & 412 & 2,2 & 195 & $1,0^{*}$ & 0,637 & 432 & 2,2 & 207 & $1,0^{*}$ & 0,643 \\
\hline \multirow{4}{*}{ TOTAL } & PE & 16 & 1,6 & 35 & 3,5 & 0,619 & 26 & 2,2 & 42 & 3,5 & 0,668 \\
\hline & MP & 37 & 1,6 & 131 & $5,8^{*}$ & 0,426 & 32 & 1,3 & 138 & $5,5^{*}$ & 0,363 \\
\hline & PM & 824 & 3,1 & 1012 & $3,8^{*}$ & $0,894^{* \text { * }}$ & 961 & 3,3 & 1003 & $3,4^{*}$ & $0,916^{\text {** * }}$ \\
\hline & $\mathbf{T}$ & 11754 & 3,6 & 6320 & $1,9^{*}$ & 0,692 & 12760 & 3,7 & 6842 & $2,0^{*}$ & 0,690 \\
\hline
\end{tabular}

${ }^{*}$ Diferencias estadísticamente significativas entre la referencia e INTERGROWTH-21 ${ }^{\text {st }}(\mathrm{p}<0,001)$.

** Concordancias muy buenas (Kappa > 0,80). EG: edad gestacional. SD: sin datos.

CABA: Ciudad Autónoma de Buenos Aires. NOA: Noroeste Argentino. NEA: Noreste Argentino. PE: prematuro extremo.

MP: muy prematuro. PM: prematuro moderado. T: término. 
relativo de la prevalencia de BP en EG tempranas de, aproximadamente, 1,2 a 3,6 veces comparado con la referencia poblacional argentina de Urquía. Para PEG, el aumento fue menor (1,12,0 veces). Por el contrario, en $\mathrm{RN}$ a término, las prevalencias fueron 1,9 veces mayores con la referencia comparada con INTERGROWTH- $21^{\text {st }}$. Estas diferencias pueden haber ocurrido porque los nacimientos pretérmino patológicos están sobrerrepresentados en las referencias. La adopción de INTERGROWTH-2 $1^{\text {st }}$ conduciría a que un número significativo de fetos fuera diagnosticado con tamaño fetal pequeño, particularmente, en pretérminos.

TABla 2: Prevalencias de pequeños para la edad gestacional con la referencia poblacional argentina de Urquía y el estándar INTERGROWTH-21 ${ }^{\text {st }}$ por sexo y categorías de edad gestacional según regiones de Argentina (2013)

\begin{tabular}{|c|c|c|c|c|c|c|c|c|c|c|c|}
\hline \multirow{3}{*}{$\begin{array}{c}\text { Región/ } \\
\text { país }\end{array}$} & \multirow{3}{*}{$\begin{array}{c}\text { Catego } \\
\text { rías } \\
\text { EG }\end{array}$} & \multicolumn{5}{|c|}{ Mujeres } & \multicolumn{5}{|c|}{ Varones } \\
\hline & & \multicolumn{2}{|c|}{ Referencia } & \multicolumn{2}{|c|}{ Estándar } & \multirow{2}{*}{ Kappa } & \multicolumn{2}{|c|}{ Referencia } & \multicolumn{2}{|c|}{ Estándar } & \multirow{2}{*}{ Kappa } \\
\hline & & $\mathbf{N}$ & Prev. & $\mathbf{N}$ & Prev. & & $\mathbf{N}$ & Prev. & $\mathbf{N}$ & Prev. & \\
\hline \multirow{4}{*}{ NOA } & PE & 10 & 7,3 & 9 & 6,6 & 0,717 & 20 & 14,8 & 13 & 9,6 & 0,8 \\
\hline & MP & 20 & 7,3 & 45 & $16,4^{*}$ & 0,572 & 35 & 12,6 & 46 & 16,6 & $0,8^{\star *}$ \\
\hline & PM & 383 & 10,4 & 418 & 11,3 & $0,951^{* *}$ & 436 & 11,4 & 411 & 10,7 & $0,9^{* *}$ \\
\hline & $\mathrm{T}$ & 3940 & 8,8 & 1975 & $4,4^{*}$ & 0,647 & 4176 & 9,1 & 2255 & $4,9^{*}$ & 0,7 \\
\hline \multirow{4}{*}{ NEA } & PE & 2 & 1,9 & 11 & $10,7^{*}$ & 0,284 & 7 & 5,9 & 7 & 5,9 & $0,848^{* *}$ \\
\hline & MP & 14 & 6,1 & 30 & $13,2^{*}$ & 0,603 & 17 & 6,9 & 29 & 11,7 & 0,714 \\
\hline & PM & 287 & 9,6 & 322 & 10,7 & $0,936^{\star * *}$ & 307 & 10,1 & 296 & 9,7 & $0,925^{* *}$ \\
\hline & $\mathrm{T}$ & 3624 & 10,7 & 2042 & $6,0^{*}$ & 0,697 & 3625 & 10,2 & 2069 & $5,8^{*}$ & 0,705 \\
\hline \multirow{4}{*}{ CUYO } & PE & 2 & 2,4 & 6 & 7,3 & 0,481 & 8 & 8,6 & 6 & 6,5 & $0,846^{\text {** }}$ \\
\hline & MP & 8 & 5,3 & 16 & 10,7 & 0,641 & 14 & 8,2 & 17 & 10,0 & $0,894^{\text {***}}$ \\
\hline & PM & 187 & 11,9 & 204 & 12,9 & $0,950^{* *}$ & 182 & 11,4 & 171 & 10,7 & $0,946^{* *}$ \\
\hline & $\mathrm{T}$ & 2577 & 9,7 & 1287 & $4,9^{*}$ & 0,643 & 2469 & 9,2 & 1311 & $4,9^{*}$ & 0,673 \\
\hline \multirow{4}{*}{ CENTRO } & PE & 38 & 6,7 & 44 & 7,8 & 0,685 & 84 & 12,2 & 57 & $8,3^{*}$ & 0,772 \\
\hline & MP & 87 & 6,6 & 173 & $13,1^{*}$ & 0,637 & 134 & 9,1 & 188 & $12,7^{*}$ & $0,812^{\text {** }}$ \\
\hline & PM & 1377 & 8,8 & 1490 & $9,5^{*}$ & $0,955^{\star *}$ & 1784 & 10,2 & 1738 & 9,9 & $0,930^{\text {** }}$ \\
\hline & $\mathrm{T}$ & 18174 & 9,9 & 9828 & $5,3^{*}$ & 0,680 & 19296 & 9,8 & 10990 & $5,6^{*}$ & 0,705 \\
\hline \multirow{4}{*}{ САBA } & $\mathrm{PE}$ & 1 & 1,6 & 5 & 8,2 & 0,315 & 16 & 17,8 & 10 & 11,1 & 0,733 \\
\hline & MP & 14 & 9,3 & 25 & 16,6 & 0,680 & 16 & 9,4 & 20 & 11,8 & $0,876^{\star * *}$ \\
\hline & PM & 145 & 10,3 & 154 & 10,9 & $0,966^{\star *}$ & 160 & 10,7 & 148 & 9,9 & $0,906^{\star *}$ \\
\hline & $\mathrm{T}$ & 1570 & 8,2 & 782 & $4,1^{*}$ & 0,646 & 1555 & 7,7 & 809 & $4,0^{*}$ & 0,667 \\
\hline \multirow{4}{*}{$\begin{array}{l}\text { PATA- } \\
\text { GONIA }\end{array}$} & PE & 4 & 6,7 & 6 & 10,0 & 0,565 & 5 & 6,3 & 3 & 3,8 & 0,738 \\
\hline & MP & 6 & 4,7 & 18 & $14,2^{*}$ & 0,462 & 15 & 8,2 & 26 & 14,1 & 0,701 \\
\hline & PM & 100 & 7,1 & 112 & 7,9 & $0,939^{* *}$ & 102 & 6,3 & 98 & 6,1 & $0,947^{* *}$ \\
\hline & $\mathrm{T}$ & 1268 & 6,8 & 632 & $3,4^{*}$ & 0,649 & 1332 & 6,8 & 687 & $3,5^{*}$ & 0,665 \\
\hline \multirow{4}{*}{ TOTAL } & PE & 57 & 5,6 & 81 & $8^{*}$ & 0,612 & 140 & 11,6 & 96 & 8 & 0,775 \\
\hline & MP & 149 & 6,6 & 307 & 13,6 & 0,620 & 231 & 9,1 & 326 & 12,9 & $0,809^{* *}$ \\
\hline & $\mathbf{P M}$ & 2479 & 9,2 & 2700 & 10,1 & $0,952^{* *}$ & 2971 & 10,2 & 2862 & $9,8^{*}$ & $0,927^{* *}$ \\
\hline & $\mathbf{T}$ & 31153 & 9,5 & 16546 & 5,1 & 0,672 & 32453 & 9,4 & 18121 & 5,2 & 0,696 \\
\hline
\end{tabular}

* Diferencias estadísticamente significativas entre la referencia e INTERGROWTH-21 ${ }^{\text {st }}(\mathrm{p}<0,001)$.

** Concordancias muy buenas (Kappa > 0,80). EG: edad gestacional. CABA: Ciudad Autónoma de Buenos Aires.

NOA: Noroeste Argentino. NEA: Noreste Argentino. PE: prematuro extremo. MP: muy prematuro. PM: prematuro moderado.

$\mathrm{T}$ : término. 
En contraste con la población altamente seleccionada de INTERGROWTH- $21^{\text {st }}$, es esperable que la población argentina tenga mayores exposiciones a factores asociados al estilo de vida, patologías obstétricas y cesáreas electivas que contribuyen a los nacimientos prematuros y restricción del crecimiento fetal. Debido a que los RN prétermino y PEG tienen el mayor riesgo de mortalidad neonatal e infantil ${ }^{14}$ es crítico identificarlos para la prevención secundaria y terciaria de discapacidad y mortalidad.

La prevalencia de PEG en RN de América Latina y el Caribe en 2010 fue 12,5\% (IC 9,416,3) y, en Argentina, 11,3\% (IC 8,2-15), de los cuales el $85 \%$, aproximadamente, eran a término. ${ }^{15}$ En el presente trabajo y a nivel regional, independientemente de la EG, la prevalencia de PEG es mayor con la referencia que con el estándar, y oscila con la referencia entre $20 \%$ (Cuyo) y 13,3\% (Patagonia) y con el estándar de 15,5\% (Cuyo) a 10,9\% (Patagonia). Este hallazgo puede atribuirse a que los PEG más pesados bajo la referencia son recategorizados a peso adecuado bajo el nuevo estándar. El punto de corte de PEG menor del P10 utilizando la referencia puede haber sido demasiado inclusivo en la identificación de neonatos en riesgo de restricción del crecimiento fetal (Figura 3). ${ }^{16}$
Las diferencias con la literatura pueden atribuirse a que, para calcular la prevalencia de PEG, en el estudio de Lee et al., ${ }^{15}$ se utilizó la referencia de Alexander et al., ${ }^{16}$ que empleaba, además de procedimientos de alisamiento de percentilos no linear, una técnica para identificar y excluir casos con incompatibilidad biológica entre PN y EG. Hasta la semana $37^{+6}$, el P10 de esta referencia era, en ambos sexos, más alto que los de la referencia poblacional argentina de Urquía y el estándar. Por esta razón, Kozuki et al., ${ }^{17}$ encontraron un relativo descenso de la prevalencia de PEG en niños pretérmino con respecto a los nacidos a término en Estados Unidos cuando se realizó la comparación entre INTERGROWTH-21 $1^{\text {st }}$ y la referencia de Alexander, ${ }^{16}$ lo que concordó con nuestro estudio.

El patrón de divergencia entre INTERGROWTH-21 ${ }^{\text {st }}$ y la referencia poblacional argentina de Urquía difiere según la EG. Los PN de RN a término con INTERGROWTH$21^{\text {st }}$ eran inferiores a los de la referencia, pero la diferencia entre las dos curvas se amplía en las EG superiores. Se especula que esta divergencia puede deberse a factores de riesgo (diabetes gestacional, sobrepeso y obesidad materna) vinculados a un mayor peso al nacer y parto prematuro, ${ }^{18}$ más prevalentes en la

FiguRa 3: Prevalencia (\%) general de bajo peso al nacer, bajo peso y pequeños para edad gestacional según la referencia poblacional argentina de Urquía y el estándar INTERGROWTH-21 $1^{\text {st }}$ por regiones en recién nacidos a término y pretérmino (Argentina, 2013)

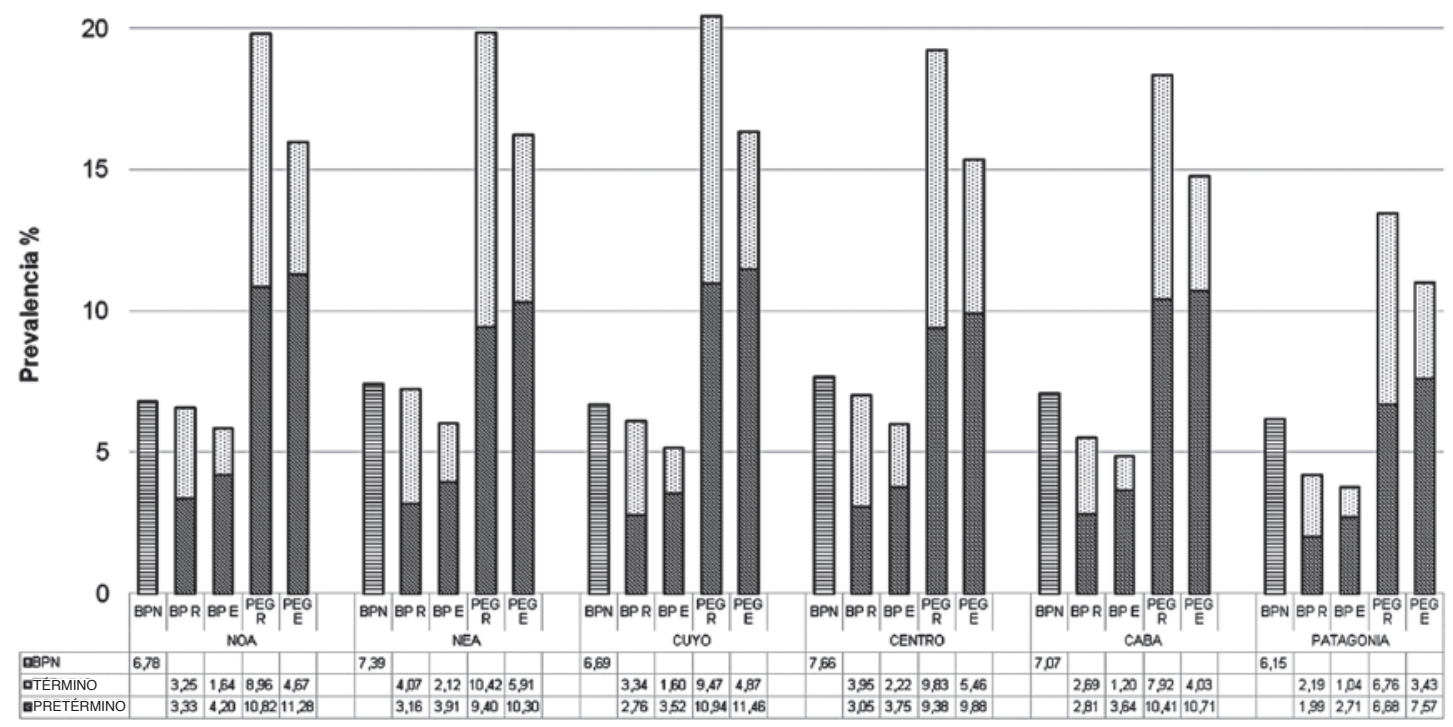

BPN: bajo peso al nacer. BP R: bajo peso, referencia poblacional argentina de Urquía. BP E: bajo peso, estándar INTERGROWTH-21 $1^{\text {st }}$ PEG R: pequeño para la edad gestacional, referencia poblacional argentina de Urquía. PEG E: pequeño para la edad gestacional, estándar INTERGROWTH-21 ${ }^{\text {st }}$. NOA: Noroeste Argentino. NEA: Noreste Argentino. CABA: Ciudad Autónoma de Buenos Aires. 
población argentina, pero excluidos del estudio INTERGROWTH-21 ${ }^{\text {st. }}$.

En RN a término, la categoría BP refleja la masa corporal relativa a la EG y es influenciada tanto por la talla como por el peso. Su interpretación resulta compleja porque puede ser determinada indistintamente o combinadamente por el acortamiento y la emaciación. No existe mucha información sobre la prevalencia de los fenotipos de malnutrición (emaciados, BP y acortado) en niños a término y, menos aún, en pretérminos. ${ }^{19}$

La prevalencia global y regional (América Latina y el Caribe) de BP en niños de 0-5 meses es de $7 \%$ y $1 \%$, respectivamente. ${ }^{20}$ Si bien las prevalencias de BP calculadas en este trabajo son mayores a las establecidas por la Encuesta Nacional de Nutrición y Salud (ENNyS) en 2004-2005, ${ }^{21}$ ambas son inferiores a los puntos de significancia clínica y epidemiológica $(10 \%)$ propuestos por la OMS. ${ }^{22}$ Con INTERGROWTH$21^{\text {st }}$, las prevalencias de BP a nivel regional son más altas, casi el doble, en los pretérminos que las observadas en los nacidos a término. Por el contrario, con la referencia, la prevalencia de BP tiende a ser más alta en los $\mathrm{RN}$ a término en la mayoría de las regiones (Figura 3).

A nivel país y en todas las regiones geográficas, se observa una concordancia muy buena de las prevalencias de BP y PEG entre la referencia y el estándar en la categoría prematuro de moderado a tardío (Tablas 1 y 2), que coincide con la superposición de las curvas de percentilos observada en la Figura 1. Sin embargo, en las restantes categorías de prematurez, las concordancias son de débiles a moderadas, razón por la cual los resultados obtenidos con el estándar y la referencia no son equivalentes. Probables explicaciones serían diferente estimación de la EG, registro de muertes fetales de corta gestación como RN vivos en la referencia en la que el retardo del crecimiento es una de sus causas ${ }^{23}$ y criterio prescriptivo de INTERGROWTH-21 ${ }^{\text {st }}$.

Independientemente del enfoque o los criterios prescriptivos, maternos y fetales utilizados por INTERGROWTH-21 ${ }^{\text {st } 6,24}$ para producir los estándares de crecimiento para fetos y $\mathrm{RN}$ siguiendo las orientaciones del Multicentre Growth Reference Study, ${ }^{25}$ el punto más crítico para la construcción de estas tablas de crecimiento longitudinal es la EG. En INTERGROWTH-21'st, la EG fue estimada por la fecha de la última menstruación (FUM) confirmada por ultrasonido precoz $\left(<14^{+0}\right.$ semanas). Si la diferencia entre US y FUM era $\leq 7$ días, la FUM era considerada válida y adoptada como la verdadera fecha biológica. Las embarazadas con diferencias $>7$ días fueron excluidas del estudio. Por el contrario, la referencia poblacional argentina de Urquía se basa en la EG estimada de acuerdo con las normas del Informe Estadístico de Recién Nacido Vivo, que emplea la FUM con un rango de $20^{+0}->42^{+6}$ semanas. ${ }^{11}$ En la referencia poblacional argentina de Urquía, los errores en la clasificación de la EG por FUM fueron corregidos con el uso de modelos de distribuciones normales mixtas ajustado por altura sobre el nivel del mar de la residencia materna ponderado por la probabilidad de que el PN perteneciera a la distribución predominante. ${ }^{9}$ Además, la referencia incluye los nacimientos gemelares y aquellos factores de riesgo asociados con la restricción del crecimiento fetal y parto prematuro. Debido a estas diferencias, el estudio INTERGROWTH-2 $1^{\text {st }}$ tuvo pocos $\mathrm{RN}$ pretérmino o postérmino y, consecuentemente, pocos $\mathrm{RN}$ aportaron datos a las EG más bajas.

Otro de los factores que pueden haber influido en las diferencias de las prevalencias de PEG y BP entre la referencia y el estándar es el tamaño de las muestras. Las muestras pequeñas afectan la estimación de los percentilos en las EG extremas. ${ }^{9}$ En el estándar INTERGROWTH-21 ${ }^{\text {st }}$, solo se seleccionaron el 35\% ( $n=20$ 488) de las embarazadas debido a los criterios de elegibilidad, mientras que la referencia poblacional argentina de Urquía se basó en 3478286 nacimientos. Esto se refleja en las Figuras 1 y 2, en las que las mayores diferencias percentilares entre la referencia y el estándar se presentaron en las EG extremas.

Los métodos para calcular los percentilos y suavizarlos también influyen en las diferencias observadas entre el estándar y la referencia. En la referencia poblacional argentina de Urquía, los percentiles se calcularon mediante la regresión de cuantiles, en tanto que, en el estándar, se utilizaron polinomios fraccionales que suponían una distribución $t$ sesgada, y diferían también en los procedimientos de suavizamiento (smoothing). 6,9

La prevalencia regional del BPN varió muy poco entre regiones, de $6,1 \%$ (Patagonia) a 7,6\% (Centro), y las menos desarrolladas del NOA y NEA presentaron valores semejantes a las más desarrolladas del centro y sur del país. Por el contrario, las prevalencias de BP y PEG exhiben una mayor variabilidad interregional. En 2013, la 
tasa de mortalidad infantil (TMI) de Argentina fue de 10,8\%; a nivel regional, la TMI osciló entre 12,6\%o (NOA) y 8,9\%o (Centro). La distribución regional de PEG y BP calculados con el estándar y la referencia guardan relación con la distribución de la TMI a nivel regional, con valores más altos en las regiones del norte del país, y refuerzan la noción de que los neonatos PEG y con BP tienen mayores riesgos de mortalidad neonatal y posneonatal, en comparación con los nacidos con un PN acorde a la EG. Este riesgo aumenta en los neonatos PEG pretérminos. ${ }^{14,26} \mathrm{La}$ incorporación a nivel epidemiológico de BP y PEG, junto con otros indicadores, como el BPN, para evaluar el riesgo de muerte infantil y resultados adversos en salud permitiría alcanzar mejores resultados en las políticas destinadas a mejorar la salud infantil.

Una probable explicación de por qué los prematuros de moderados a tardíos fueron los únicos con elevado Kappa es su mayor prevalencia entre los pretérmino (87\% entre 2003 y 2013) $)^{11}$ y la otra es su comportamiento en la morbimortalidad (especialmente, entre 35 y 36 semanas), de forma parecida a los de término temprano (37-38 semanas). ${ }^{27}$

La principal limitación de este estudio fue la falta de datos, a los fines comparativos, de las prevalencias de PEG y BP a nivel regional, mientras que la principal fortaleza fue que incluyó todos los nacimientos vivos de Argentina en 2013.

\section{CONCLUSIONES}

Las concordancias de las prevalencias BP y PEG obtenidas con el estándar INTERGROWTH$21^{\text {st }}$ y la referencia poblacional argentina de Urquía en RN pretérmino y a término fueron moderadas, y se observó variabilidad interregional. Los resultados plantean nuevas perspectivas auxológicas en la evaluación epidemiológica de la restricción del crecimiento intrauterino en Argentina. Sin embargo, la adopción de la referencia y el estándar para estudios epidemiológicos requiere su validación como indicadores de morbilidad y mortalidad en RN pretérmino y a término.

\section{REFERENCIAS}

1. World Health Organization, Expert Committee on Maternal and Child Health. Public health aspects of low birth weight: Third report of the Expert Committee on Maternal and Child Health. Geneva: World Health Organization,1961;217:3. [Acceso: 1 dejunio de 2017]. Disponibleen:http:/ / apps.who. int/iris/bitstream/10665/40487/1/WHO_TRS_217.pdf.

2. World Health Organization. WHA Global nutrition targets 2025: low birth weight policy brief. Geneva: WHO, 2014.
[Acceso: 1 de junio de 2017]. Disponible en: http: / / www. who.int/nutrition/topics/globaltargets_lowbirthweight_ policybrief.pdf.

3. Christian P, Lee SE, Donahue AM, et al. Risk of childhood undernutrition related to small-for-gestational age and preterm birth in low- and middle-income countries. Int J Epidemiol 2013;42(5):1340-55.

4. Barker DJ, Osmond C, Golding J, et al. Growth in utero, blood pressure in childhood and adult life, and mortality from cardiovascular disease. BMJ 1989;298(6673):564-7.

5. Comité de Crecimiento y Desarrollo y Comité de Estudios Fetoneonatales (CEFEN). Propuesta de Actualización de la Evaluación Antropométrica del Recién Nacido. Arch Argent Pediatr 2017;115(1):89-95.

6. Villar J, Cheikh IL, Victora CG, et al. International standards for newborn weight, length, and head circumference by gestational age and sex: the Newborn Cross-Sectional Study of the INTERGROWTH-21 ${ }^{\text {st }}$ Project. Lancet 2014;384(9946):857-68.

7. De Onis M, Garza C, Onyango A, et al edit. WHO child growth standards. Acta Paediatrica 2006;95(Suppl450):1-104.

8. Comité Nacional de Crecimiento y Desarrollo. Guía para la evaluación del crecimiento físico. 3. ${ }^{\text {ra }}$ ed. Buenos Aires: Sociedad Argentina de Pediatría; 2013.

9. Urquia ML, Alazraqui M, Spinelli HG, et al. Referencias poblacionales argentinas de peso al nacer según multiplicidad del parto, sexo y edad gestaciona. Rev Panam Salud Pública 2011;29(2):108-19.

10. Villar J, Giuliani F, Fenton TR, et al. INTERGROWTH$21^{\text {st }}$ very preterm size at birth reference charts. Lancet 2016;387(10021):844-5.

11. Argentina. Ministerio de Salud. Dirección de Estadísticas e Información de Salud. Estadísticas vitales. [Acceso: 21 de octubre de 2016]. Disponible en http:/ / www.deis.msal. gov.ar/index.php/estadisticas-vitales/.

12. Organización Mundial de la Salud. Nacimientos prematuros, 2016. [Acceso: 10 de diciembre de 2016]. Disponible en: http://www.who.int/mediacentre/ factsheets/fs363/es/.

13. LandisJR, KochGG. Themeasurement of observer agreement for categorical data. Biometrics 1977;33(1):159-74.

14. Katz J, Lee AC, Kozuki N, et al. Mortality risk in preterm and small-for-gestational-age infants in low-income and middle-income countries: a pooled country analysis. Lancet 2013;382(9890):417-25.

15. Lee AC, Katz J, Blencowe $\mathrm{H}$, et al. National and regional estimates of term and preterm babies born small for gestational age in 138 low-income and middle-income countries in 2010. Lancet Glob Health 2013;1(1):e26-36.

16. Alexander GR, Himes JH, Kaufman RB, et al. A United States national reference for fetal growth. Obstet Gynecol 1996;87(2):163-8.

17. Kozuki N, Katz J, Christian P, et al. Comparison of US Birth weight References and the International Fetal and Newborn Growth Consortium for the $21^{\text {st }}$ Century Standard. JAMA Pediatr 2015;169(7):e151438.

18. Yu Z, Han S, Zhu J, et al. Pre-pregnancy body mass index in relation to infant birth weight and offspring overweight/ obesity: a systematic review and meta-analysis. PLoS One 2013;8(4):e61627.

19. Victora C, Villar J, Barros F, et al. Anthropometric Characterization of Impaired Fetal Growth: Risk Factors for and Prognosis of Newborns with Stunting or Wasting. JAMA Pediatr 2015;169(7):e151431.

20. Lopriore C, Dop MC, Solal-Céligny A, et al. Excluding infants under 6 months of age from surveys: impact on prevalence of pre-school undernutrition. Public Health Nutr 2007;10(1):79-87. 
21. Durán P, Mangialavori G, Biglieri A, et al. Estudio descriptivo de la situación nutricional en niños de 6-72 meses de la República Argentina. Resultados de la Encuesta Nacional de Nutrición y Salud (ENNyS). Arch Argent Pediatr 2009;107(5):397-404.

22. World Health Organization. Physical Status: The use and interpretation of anthropometry. Geneva: World Health Organization; 1995. [Acceso: 1 dejunio de 2017]. Disponible en:http: / / apps.who.int/iris/bitstream/10665/37003/1/ WHO_TRS_854.pdf.

23. Lawn JE, Blencowe $H$, Waiswa $P$, et al. Stillbirths: rates, risk factors, and acceleration towards 2030. Lancet 2016;387(10018):587-603.
24. Villar J, Altman DG, Purwar M, et al. The objectives, design and implementation of the INTERGROWTH-21 ${ }^{\text {st }}$ Project. BJOG 2013;120(Suppl 2):9-26.

25. De Onis M, Onyango AW, Van den Broeck J, et al. Measurement and standardization protocols for anthropometry used in the construction of a new international growth reference. Food Nutr Bull 2004;25(Suppl 1):S27-36.

26. Grisaru-Granovsky S, Reichman B, Lerner-Geva L, et al. Mortality and morbidity in preterm small-for-gestationalage infants: a population-based study. Am J Obstet Gynecol 2012;206(2):150:e1-7.

27. Engle W, Tomashek K, Wallman C, et al. "Late-Preterm" Infants: a population at risk. Pediatrics 2007;120(6):1390-401.

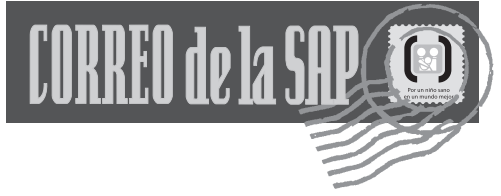

Los siguientes resúmenes y comentarios de trabajos seleccionados se encuentran disponibles en la versión electrónica de este número.

PEDIATRICS 2017;139(5):e20163403

Síntomas virales y pesquisa de faringitis estreptocóccica (Shapiro DJ, et al. Viral Features and Testing for Streptococcal Pharyngitis)

Comentario: Dr. Juan Bautista Dartiguelongue. Hospital de Niños Ricardo Gutiérrez. Buenos Aires.

PEDIATRICS 2017 Apr;139(4)

Lesiones relacionadas con productos infantiles tratadas en los departamentos de emergencias de Estados Unidos (Gaw CE, et al. Nursery Product-Related Injuries Treated in United States Emergency Departments)

Comentario: Dra. Ingrid Waisman. Neoclínica. Río Cuarto, Córdoba.

CHILD DEV 2017 May 15. doi: 10.1111/cdev.12827. [Epub ahead of print]

Distracciones al caminar, circular en bicicleta o conducir: revisión sistemática y meta-análisis de tecnología móvil y riesgo de accidente en jóvenes (Stavrinos D, et al. Distracted walking, bicycling, and driving: systematic review and meta-analysis of mobile technology and youth crash risk)

Comentario: Dra. Laura Krynski. Subcomisión de Tecnologías de Información y Comunicación SAP.

INTERNATIONAL JOURNAL OF OBESITY (2017), 1-7.

Asociaciones longitudinales entre la televisión en el dormitorio y la obesidad, estudio de cohortes del Reino Unido (Heilmann A, et al. Longitudinal associations between television in the bedroom and body fatness in a UK cohort study)

Comentario: Dr. Alberto Hernández. Comité de Historia Clínica Informatizada. Hospital de Pediatría "Prof. Dr. Juan P. Garrahan", Buenos Aires.

LANCET 2017 Jun 17;389(10087):2393-2402. Epub 2017 Apr 11.

Precisión de las reglas de decisión sobre traumatismo de cráneo en niños, PECARN, CATCH y CHALICE: un estudio de cohorte prospectivo (Babl FE, et al. Accuracy of PECARN, CATCH, and CHALICE head injury decision rules in children: a prospective cohort study)

Comentario: Dra. Gabriela Hualde. Servicio de Emergencias. Hospital de Pediatría “Prof. Dr. Juan P. Garrahan", Buenos Aires. 